\title{
Domain decomposition preconditioners for multiscale flows in high contrast media
}

\author{
Juan Galvis* $\quad$ Yalchin Efendiev ${ }^{\dagger}$
}

\begin{abstract}
In this paper, we study domain decomposition preconditioners for multiscale flows in high contrast media. Our problems are motivated by porous media applications where low conductivity regions play an important role in determining flow patterns. We consider flow equations governed by elliptic equations in heterogeneous media with large contrast between high and low conductivity regions. This contrast brings an additional small scale (in addition to small spatial scales) into the problem expressed as the ratio between low and high conductivity values. Using weighted coarse interpolation, we show that the condition number of the preconditioned systems using domain decomposition methods is independent of the contrast. For this purpose, Poincaré inequalities for weighted norms are proved in the paper. The results are further generalized by employing extension theorems from homogenization theory. Our numerical observations confirm the theoretical results.
\end{abstract}

\section{Introduction}

Subsurface flows are often affected by heterogeneities in a wide range of length scales. Moreover, the media properties often vary significantly that introduces an additional level of complexity. A high contrast in the media properties brings an additional small scale into the problem expressed as the ratio between low and high conductivity values. For example, it is very common to have several orders of magnitude of variations in the permeability field in natural porous formations. In addition, low conductivity regions can have complex geometry that can introduce connected barriers at very small scales in the flow.

In this paper, we study efficient techniques for solving flows in highly heterogeneous formations. It is common to use upscaled or multiscale models to solve flow and transport processes on the coarse grid [1, 2, 4, 5, 6, 7, 8, 11, 23].

\footnotetext{
*Department of Mathematics, Texas A \& M University, College Station, TX 77843 (jugal@math.tamu.edu)

${ }^{\dagger}$ Department of Mathematics, Texas A \& M University, College Station, TX 77843 (efendiev@math.tamu.edu)
} 
These approaches approximate the effects of the fine-scale features and attempt to capture these effects on a coarse grid via localized basis functions. In many cases, multiscale approaches can not provide an accurate approximation of the solution and one needs to solve for the fine-scale solution. The solution of the fine-scale equation is typically prohibitively expensive because of the small scales and high contrast in the conductivity field. For this reason, some type of preconditioning is needed to reduce the number of iterations required for solving the fine-scale system of equations.

In this paper we investigate domain decomposition preconditioners for flows in heterogeneous porous media. Domain decomposition methods use the solutions of several local coarse problems in constructing preconditioners for the fine-scale system. The number of iterations required by domain decomposition preconditioners is typically affected by the contrast in the media properties. Our main objective is to analyze the dependence of the domain decomposition preconditioners on the media properties. In particular we will concentrate on developing tools that allow identifying the media properties where domain decomposition methods provide good results (low number of iterations) independent of the contrast of the media. It is known that if high and low conductivity regions can be encompassed within coarse grid blocks such that the variation of the conductivity within each coarse region is bounded, domain decomposition preconditioners result to a system with the condition number independent of the contrast (e.g., $[20,25])$. Because of complex geometry of fine-scale features, it is often impossible to separate low and high conductivity regions into different coarse grid blocks. Indeed, low conductivity regions can have small sizes in the shape of narrow channels. Encompassing these regions into coarse grids can significantly increase the number of coarse-grid blocks and make the computations expensive.

When the conductivity field varies significantly within each coarse-grid block, analysis of domain decomposition methods is a challenging task. In a recent pioneering work $[15,16]$, it has been shown that using domain decomposition methods, one can precondition the fine-scale system such that the condition number of the resulting system is independent of the contrast when high conductivity inclusions are embedded into the media of bounded conductivity. The approach presented in [15] is not applicable to the problems when low contrast inclusions are present in the high contrast media that we study in this paper. The heterogeneities considered in our paper require a careful analysis of domain decomposition preconditioners. In our analysis, we define a coarsescale interpolation using weighted averages. We show weighted $H^{1}$ stability and weighted $L^{2}$ approximation this coarse quasi-interpolation operator. Our analysis requires special Poincaré estimates to bound the weighted $L_{2}$ estimates. We study the heterogeneities when the low conductivity regions are embedded into high conductivity flow regions. Because low conductivity regions can change flow patterns significantly (e.g., can block the flow), the study of their effects is important in the fine-scale simulations [19, 4, 17].

We study two level additive Schwartz preconditioners with several coarse solvers. We analyze theoretically and numerically the performance of domain 
decomposition methods in situations similar to the ones encountered in subsurface applications. In the overlapping setting, since we would like to treat heterogeneous media, we concentrate on the case of generous and big overlap. In this case the size of the overlap is of the order of the size of the coarse triangulation parameter. As mention before, we employ weighted averages to construct a coarse-scale interpolation. The estimation of the energy of the local functions requires Poincaré inequalities for weighted averages that is an important part of the proof. We prove this inequality for a wide range of heterogeneities.

We also study the behavior of overlapping domain decomposition preconditioners for the Schur complement problem. The Schur complement is obtained after eliminating the subdomain interior degrees of freedoms. It is known that nonoverlapping preconditioners are well suited for piecewise constant coefficients $([20,25])$ with respect to the nonoverlaping decomposition used to construct the method. However, the analysis of these methods in the cases with significant conductivity variations within and between adjacent coarse-grid blocks is not straightforward and is still an active research topic. Instead of a nonoverlapping preconditioner, we use an overlapping two level additive method for the Schur complement problem ([3, 26]). Using this framework, we discuss how one can reduce the number of low conductivity inclusions using extension theorems $[14,9,10]$. These theorems are often used in homogenization theory [14]. This reduction can be regarded as a partial homogenization because it homogenizes many inclusions such that the resulting media has fewer inclusions where our new Poincaré estimates can be applied.

For the coarse solvers, we consider various choices for basis functions - piecewise linear basis, multiscale finite element basis functions, and energy minimizing basis functions. We find that multiscale finite element basis functions and energy minimizing basis functions result to a fewer iterations. This has been observed in previous findings, e.g., [15]. From the proof, it becomes evident that the coarse basis functions with minimum energy are needed to minimize the number of iterations.

Numerical results are presented to show that the condition number of the preconditioned system is independent of the contrast. We test our approaches on a number of examples where the low conductivity regions have various complex geometries. In all cases, we observe that the condition number of the preconditioned system is independent of the contrast. Both domain decomposition preconditioners are studied numerically.

The paper is organized as follows. In the next section, we formulate the problem. Section 3 is devoted to the motivation and summary of the results. In Section 4, we present overlapping methods and their analysis. Section 5 is devoted to overlapping methods for Schur complement. In this section, we also discuss how to reduce the number of low conductivity inclusions by partial homogenization. We make some comments on the use of domain decomposition preconditioners in two-phase flow setting in Section 6. The numerical results are presented in Section 7 . 


\section{Problem Setting}

Let $D \subset \mathbb{R}^{2}$ (or $\mathbb{R}^{3}$ ) be a polygonal domain which is the union of a disjoint polygonal subregions $\left\{D_{i}\right\}_{i=1}^{N}$. We consider the following problem. Find $u^{*} \in$ $H_{0}^{1}(D)$ such that

$$
a\left(u^{*}, v\right)=f(v) \quad \text { for all } v \in H_{0}^{1}(D) .
$$

Here the bilinear for $a$ and the linear functional $f$ are defined by

$$
a(u, v)=\int_{D} \kappa(x) \nabla u(x) \nabla v(x) d x \quad \text { for all } u, v \in H_{0}^{1}(D)
$$

and

$$
f(v)=\int_{D} f(x) v(x) d x \quad \text { for all } v \in H_{0}^{1}(D) .
$$

We assume that the decomposition $\left\{D_{i}\right\}_{i=1}^{N}$ form a quasiuniform triangulation of $D$ with parameter $H=\max _{i} \operatorname{diam}\left(D_{i}\right)$. This coarse triangulation will be also denoted by $\mathcal{T}^{H}$. Let $\mathcal{T}^{h}$ be a fine triangulation which is a refinement of $\mathcal{T}^{H}$.

We denote by $V^{h}(D)$ the usual finite element discretization of piecewise linear continuous functions with respect to the fine triangulation $\mathcal{T}^{h}$. Denote also by $V_{0}^{h}(D)$ the subset of $V^{h}(D)$ with vanishing values on $\partial D$. Similar notations, $V^{h}(\Omega)$ and $V_{0}^{h}(\Omega)$, are used for subdomains $\Omega \subset D$.

The Galerkin formulation of (1) is to find $u^{*} \in V_{0}^{h}(D)$ such that

$$
a\left(u^{*}, v\right)=f(v) \quad \text { for all } v \in V_{0}^{h}(D),
$$

or in matrix form

$$
A u^{*}=b
$$

where for all $u, v \in V^{h}(D)$ we have

$$
u^{T} A v=\int_{D} \kappa \nabla u \nabla v \quad \text { and } \quad v^{T} b=\int_{D} f v .
$$

Let $A^{(i)}$ be the local matrices corresponding to the bilinear form (2), i.e., for all $u, v \in V^{h}\left(D_{i}\right)$ we have

$$
u^{T} A^{(i)} i v=\int_{D_{i}} \kappa \nabla u \nabla v .
$$

\section{Motivation and summary of results}

Our motivation stems from flow problems that occur in heterogeneous porous formations. In flow problems, the high conductivity (permeability) regions play an important role. These high conductivity channels are the main carriers of the 
fluid. The high conductivity regions are often "polluted" by low permeability layers (shale layers) as schematically depicted in Figure 1. Low conductivity layers can occur in oil reservoirs and can introduce low permeability barriers at different scales. These low conductivity regions can have complicated connectivity. Because of complex geometry of shale layers, it is often impossible to separate them into coarse grid blocks without significantly increasing the computational effort. In this paper, our objective is to show that domain decomposition based preconditioners result to systems with a condition number independent of the contrast of the media.

Previous approaches concerning overlapping domain decomposition address the cases where the permeability fields are nearly homogeneous within each coarse-grid block (e.g., [20, 25]) or high conductivity regions constitute disconnected regions within a coarse-grid block $[15,16]$. Our proof differs from the one presented in [15] and uses weighted averages to construct a coarse-scale quasiinterpolation. To verify a stable decomposition, Poincaré estimates are proved for the weighted $L_{2}$ norms in the form $\int \kappa(x) v^{2} \leq C \int \kappa(x)|\nabla v|^{2}$, where $C$ is independent of the contrast in $\kappa(x)$. Based this new type of Poincaré inequality, we are able to show that domain decomposition based preconditioners result to a system that has condition number independent of the contrast of the media. We note that in previous approaches $[15,16]$ standard volume averages are used for the coarse-scale approximants and there is no need for special Poincaré estimates. We highlight the difference between our case and the case presented in $[15,16]$ after the proof of Lemma 8.

We recall that the behavior of domain decomposition methods for (4) with respect to the parameters of the fine and coarse triangulation is well known (e.g., $[20,25])$. We also note that only the case of generous or big overlap $\delta$ is considered here. The presence of fine-scale features makes difficult the analysis of small overlap methods in our setting. In Lemma 10 we prove the condition number for the two level preconditioner is of order $\left.O\left((H / \delta)^{2}\right)\right)$ and is independent of the contrast. Here we consider the case of a high contrast coefficient with a finite number of (low conductivity inclusions) in each coarse block. The analysis presented here can be extended to the case where some of the inclusions touch or cross the boundary of the coarse block.

We consider an overlapping method to precondition the Schur complement problem. We also are able to show that number of iterations is independent of the contrast. This is done for the same type of coefficients considered in the overlapping case. Then, we try to classify the types of heterogeneities that yield energies equivalent to a piecewise constant energy or any other given energy where this (or any similar) preconditioner performs well. In the analysis, we also employ extension theorems to reduce the number of low contrast inclusions. As for coarse-scale solvers, we consider various choices for coarse basis functions - piecewise linear basis, multiscale finite element basis functions, and energy minimizing basis functions. We find that multiscale finite element basis functions and energy minimizing basis functions result to a fewer iterations. The latter is also evident from the proof. 


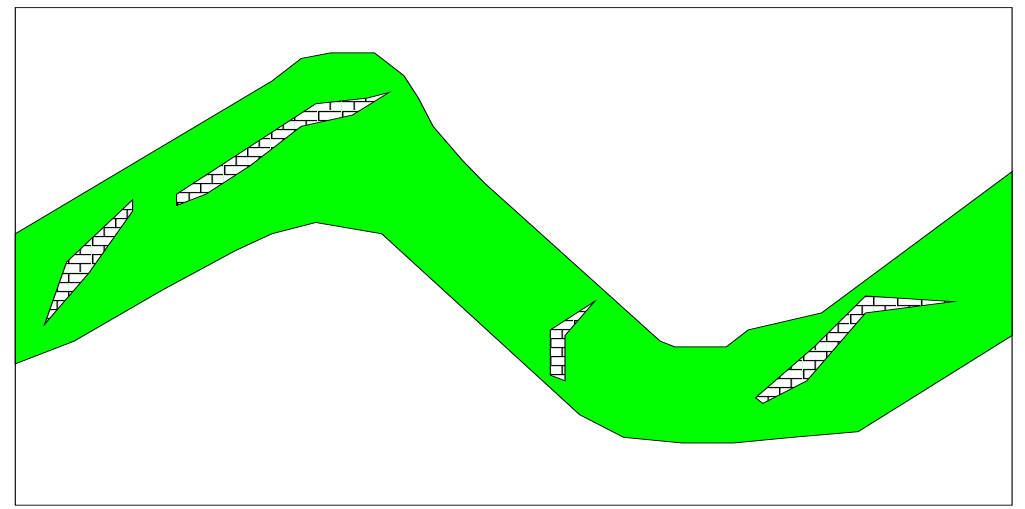

Figure 1:

\section{Overlapping methods}

\subsection{Notation}

We denote by $\left\{D_{i}^{\prime}\right\}_{i=1}^{N}$ the overlapping decomposition obtained from the original nonoverlapping decomposition $\left\{D_{i}\right\}_{i=1}^{N}$ by enlarging each subdomain $D_{i}$ to

$$
D_{i}^{\prime}=D_{i} \cup\left\{x \in D, \operatorname{dist}\left(x, D_{i}\right)<\delta\right\}, \quad i=1, \ldots, N,
$$

where dist is some distance function and let $V_{0}^{i}\left(D_{i}^{\prime}\right)$ be the set of finite element functions with support in $D_{i}^{\prime}$. We also denote by $R_{i}^{T}: V_{0}^{i}\left(D_{i}^{\prime}\right) \rightarrow V^{h}$ the extension by zero operator.

Now we introduce a coarse triangulation $\mathcal{T}^{H}$ and coarse vertex based basis functions $\left\{\Phi_{i}\right\}_{i=1}^{N_{c}}$. In the general setting of domain decomposition solvers this coarse level triangulation may be independent of the subdomain partition of the original domain $D$. In order to simplify the analysis we assume that the coarse triangulation coincides with the nonoverlapping decomposition. We also assume that the coarse basis functions satisfy:

1. $\left|\Phi_{i}\right| \preceq 1, i=1, \ldots, N_{c}$.

2. $\sum_{i=1}^{N_{c}} \Phi_{i}=1$.

Define

$$
V_{0}=\operatorname{span}\left\{\Phi_{i}\right\}_{i=1}^{N_{c}}
$$

where $\Phi_{i}, i=1, \ldots, M$, are coarse-scale finite element basis functions. We use a two level additive preconditioner of the form

$$
B^{-1}=R_{0} A_{0}^{-1} R_{0}+\sum_{i=1}^{N} R_{i}^{T} A_{i}^{-1} R_{i}
$$




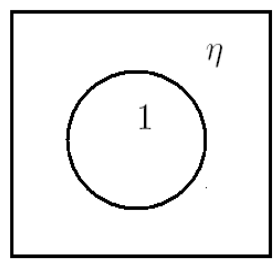

a)

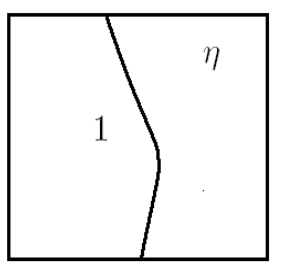

b)

Figure 2: Domain configuration in Lemma 1.

where the local matrices are defined by

$$
v A_{i} w=a(v, w) \quad \text { for all } v, w \in V_{0}^{i}\left(D_{i}^{\prime}\right), \quad i=1, \ldots, N,
$$

and the coarse matrix $A_{0}=R_{0} A R_{0}^{T}$ with $R_{0}^{T}: V^{h} \rightarrow R_{0}$ being the projection onto the coarse space $V_{0}$.

Throughout, $a \preceq b$ means that $a \leq C b$ where the constant $C$ is independent of the mesh size and contrast. In the analysis, $\Omega_{\eta}$ denote the regions with high conductivity coefficients (of order $\eta$ assuming $\eta$ is large) and $\Omega_{1}$ denote the regions with the bounded conductivity (of order 1), unless otherwise is specified.

\subsection{Some technical tools}

Lemma 1 Let $\kappa$ be defined by

$$
\kappa(x)= \begin{cases}1, & x \in \Omega_{1}, \\ \eta, & x \in \Omega_{\eta},\end{cases}
$$

where $\Omega_{1}$ and the inclusion $\Omega_{\eta}$ are Lipschitz domains; see Figure 2 a). Then, for every $v \in H^{1}(\Omega)$ such that $\int_{\Omega} \kappa v=0$ we have

$$
\int_{\Omega} \kappa v^{2} \preceq H^{2} \int_{\Omega} \kappa|\nabla v|^{2} .
$$

Proof. From the usual Poincaré inequality we have

$$
\int_{\Omega_{1}}\left|v-\bar{v}_{1}\right|^{2} \preceq H_{1}^{2} \int_{\Omega_{1}}|\nabla v|^{2}
$$

where $\bar{v}_{1}=\frac{1}{\left|\Omega_{1}\right|} \int_{\Omega_{1}} v$. This implies

$$
\int_{\Omega_{1}} v^{2} \preceq H_{1}^{2} \int_{\Omega_{1}}|\nabla v|^{2}+\left|\Omega_{1}\right| \bar{v}_{1}^{2} .
$$

Analogously

$$
\int_{\Omega_{\eta}} v^{2} \preceq H_{\eta}^{2} \int_{\Omega_{\eta}}|\nabla v|^{2}+\left|\Omega_{\eta}\right| \bar{v}_{\eta}^{2}
$$


where $\bar{v}_{\eta}=\frac{1}{\left|\Omega_{\eta}\right|} \int_{\Omega_{\eta}} v$. Then

$$
\begin{aligned}
\int_{\Omega} \kappa v^{2} & =\int_{\Omega_{1}} v^{2}+\eta \int_{\Omega_{\eta}} v^{2} \\
& \preceq H_{1}^{2} \int_{\Omega_{1}}|\nabla v|^{2}+H_{\eta}^{2} \eta \int_{\Omega_{\eta}}|\nabla v|^{2}+\left|\Omega_{1}\right| \bar{v}_{1}^{2}+\eta\left|\Omega_{\eta}\right| \bar{v}_{\eta}^{2} .
\end{aligned}
$$

Now we bound the last two terms in (11). We note that

$$
\int_{\Omega} k v=\left|\Omega_{1}\right| \bar{v}_{1}+\eta\left|\Omega_{\eta}\right| \bar{v}_{\eta}=0 \quad \text { and then } \quad \bar{v}_{1}=-\eta \frac{\left|\Omega_{\eta}\right|}{\left|\Omega_{1}\right|} \bar{v}_{\eta} .
$$

Let $\Gamma=\partial \Omega_{1}$ and $c=\frac{1}{|\Gamma|} \int_{\Gamma} v$. Using a Poincaré type inequality, see [25, Lemma A.17], we can show that

$$
\begin{aligned}
\left(\bar{v}_{1}-\bar{v}_{\eta}\right)^{2} & =\left(\frac{1}{\left|\Omega_{1}\right|} \int_{\Omega_{1}} v-\frac{1}{\left|\Omega_{\eta}\right|} \int_{\Omega_{\eta}} v\right)^{2} \\
& \preceq\left(\frac{1}{\left|\Omega_{1}\right|} \int_{\Omega_{1}} v-c\right)^{2}+\left(\frac{1}{\left|\Omega_{\eta}\right|} \int_{\Omega_{\eta}} v-c\right)^{2} \\
& \preceq\left(\frac{1}{\left|\Omega_{1}\right|^{\frac{1}{2}}}\|v-c\|_{L^{2}\left(\Omega_{1}\right)}\right)^{2}+\left(\frac{1}{\left|\Omega_{\eta}\right|^{\frac{1}{2}}}\|v-c\|^{2}\right)^{2} \\
& \preceq \frac{H^{2}}{\left|\Omega_{1}\right|} \int_{\Omega_{1}}|\nabla v|^{2}+\frac{H^{2}}{\left|\Omega_{\eta}\right|} \int_{\Omega_{\eta}}|\nabla v|^{2}
\end{aligned}
$$

which together with (12) give

$$
\left(1+\eta \frac{\left|\Omega_{\eta}\right|}{\left|\Omega_{1}\right|}\right)^{2} \bar{v}_{\eta}^{2}=\left(\bar{v}_{1}-\bar{v}_{\eta}\right)^{2} \preceq \frac{H_{1}^{2}}{\left|\Omega_{1}\right|} \int_{\Omega_{1}}|\nabla v|^{2}+\frac{H_{\eta}^{2}}{\left|\Omega_{\eta}\right|} \int_{\Omega_{\eta}}|\nabla v|^{2} .
$$

Then

$$
\eta\left|\Omega_{\eta}\right| \bar{v}_{\eta}^{2} \preceq \frac{\eta H_{1}^{2} \frac{\left|\Omega_{\eta}\right|}{\left|\Omega_{1}\right|}}{\left(1+\eta \frac{\left|\Omega_{\eta}\right|}{\left|\Omega_{1}\right|}\right)^{2}} \int_{\Omega_{1}}|\nabla v|^{2}+\frac{\eta H_{\eta}^{2}}{\left(1+\eta\left|\Omega_{\eta}\right|\right)^{2}} \int_{\Omega_{\eta}}|\nabla v|^{2} .
$$

Using (14) and (12)

$$
\begin{aligned}
\left|\Omega_{1}\right| \bar{v}_{1}^{2} & =\frac{\eta\left|\Omega_{\eta}\right|}{\left|\Omega_{1}\right|}\left(\eta\left|\Omega_{\eta}\right| \bar{v}_{\eta}^{2}\right) \\
& \preceq \frac{\eta^{2} H_{1}^{2} \frac{\left|\Omega_{\eta}\right|^{2}}{\left|\Omega_{1}\right|^{2}}}{\left(1+\eta \frac{\left|\Omega_{\eta}\right|}{\left|\Omega_{1}\right|}\right)^{2}} \int_{\Omega_{1}}|\nabla v|^{2}+\frac{\eta^{2} H_{\eta}^{2} \frac{\left|\Omega_{\eta}\right|}{\left|\Omega_{1}\right|}}{\left(1+\eta \frac{\left|\Omega_{\eta}\right|}{\left|\Omega_{1}\right|}\right)^{2}} \int_{\Omega_{\eta}}|\nabla v|^{2} .
\end{aligned}
$$


With (14) and (15) we get

$$
\begin{aligned}
\left|\Omega_{1}\right| \bar{v}_{1}^{2}+\eta\left|\Omega_{\eta}\right| \bar{v}_{\eta}^{2} & \preceq \frac{\eta \frac{\left|\Omega_{\eta}\right|}{\left|\Omega_{1}\right|}+\eta^{2} \frac{\left|\Omega_{\eta}\right|^{2}}{\left|\Omega_{1}\right|^{2}}}{\left(1+\eta \frac{\Omega_{\eta} \mid}{\left|\Omega_{1}\right|}\right)^{2}} H_{1}^{2} \int_{\Omega_{1}}|\nabla v|^{2}+\frac{1+\eta \frac{\left|\Omega_{\eta}\right|}{\left|\Omega_{1}\right|}}{\left(1+\eta \frac{\left|\Omega_{\eta}\right|}{\left|\Omega_{1}\right|}\right)^{2}} \eta H_{\eta}^{2} \int_{\Omega_{\eta}}|\nabla v|^{2} \\
& \leq H_{1}^{2} \int_{\Omega_{1}}|\nabla v|^{2}+\eta H_{\eta}^{2} \int_{\Omega_{\eta}}|\nabla v|^{2} .
\end{aligned}
$$

Putting (16) into (11) we get,

$$
\int_{\Omega} \kappa v^{2} \preceq 2 H_{1}^{2} \int_{\Omega_{1}}|\nabla v|^{2}+2 H_{\eta}^{2} \eta \int_{\Omega_{\eta}}|\nabla v|^{2} .
$$

Remark 2 We note that Lemma 1 holds for any inclusion $\Omega_{\eta}$ that has Poincaré property (such that (10) and (9) hold). This also holds true for the case of Figure $2 b)$.

Remark 3 In Lemma 1, the coefficient $\kappa$ is piecewise constant. The result can be easily extended to piecewise smooth coefficients such that the coefficients are of order $\eta$ or 1 in respective regions.

Remark 4 We note that Lemma 1 holds if one interchanges high- and lowconductivity regions. Proof remains the same in this case.

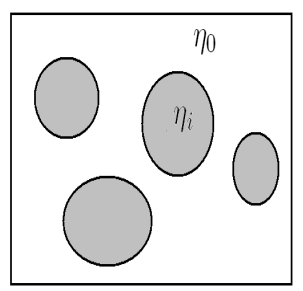

Figure 3: Domain configuration in Lemma 5.

Lemma 5 Let $\kappa$ be defined by

$$
\kappa(x)=\left\{\begin{array}{lc}
\eta_{i}, & x \in \Omega_{i}, \\
\eta_{0}, & x \in \Omega_{0}=\Omega \backslash \cup_{j=1}^{M} \Omega_{i},
\end{array}\right.
$$

where $\Omega_{i}, i=0, \ldots, M$ are Lipschitz domains; see Figure 3. Assume also that

$$
\eta_{i}\left|\Omega_{i}\right| \preceq \eta_{0}\left|\Omega_{0}\right|, \quad i=1, \ldots, M .
$$


Then, for every $v \in H^{1}(\Omega)$ such that $\int_{\Omega} \kappa v=0$ we have

$$
\int_{\Omega} \kappa v^{2} \preceq M H^{2} \int_{\Omega} \kappa|\nabla v|^{2} .
$$

Proof. From the usual Poincaré inequality we have

$$
\int_{\Omega_{0}}\left|v-\bar{v}_{0}\right|^{2} \preceq H^{2} \int_{\Omega_{0}}|\nabla v|^{2}
$$

where $\bar{v}_{0}=\frac{1}{\left|\Omega_{0}\right|} \int_{\Omega_{0}} v$. This implies

$$
\int_{\Omega_{0}} v^{2} \preceq H^{2} \int_{\Omega_{0}}|\nabla v|^{2}+\left|\Omega_{0}\right| \bar{v}_{0}^{2} .
$$

Analogously, for $i=1, \ldots, M$,

$$
\int_{\Omega_{i}} v^{2} \preceq H_{i}^{2} \int_{\Omega_{i}}|\nabla v|^{2}+\left|\Omega_{i}\right| \bar{v}_{i}^{2}
$$

where $\bar{v}_{i}=\frac{1}{\left|\Omega_{i}\right|} \int_{\partial \Omega_{i}} v, i=1, \ldots, M$. Then

$$
\int_{\Omega} \kappa v^{2}=\sum_{i=0}^{M} \eta_{i} \int_{\Omega_{i}} v^{2} \preceq \sum_{i=0}^{M} \eta_{i} H_{i}^{2} \int_{\Omega_{i}}|\nabla v|^{2}+\sum_{i=0}^{M} \eta_{i}\left|\Omega_{i}\right| \bar{v}_{i}^{2} .
$$

Now we bound the last sum in (20). We note that

$$
\int_{\Omega} k v=\sum_{i=0}^{M} \eta_{i}\left|\Omega_{i}\right| \bar{v}_{i}=0 \quad \text { and then } \quad \eta_{0}\left|\Omega_{0}\right| \bar{v}_{0}=-\sum_{i=1}^{M} \eta_{i}\left|\Omega_{i}\right| \bar{v}_{i}
$$

Let $\Gamma_{i}=\partial \Omega_{i}$ and $c_{i}=\frac{1}{\left|\Gamma_{i}\right|} \int_{\Gamma_{i}} v, i=1, \ldots, M$. Using a Poincaré inequality and the same argument to get (13) in the proof of Lemma 1 we obtain

$$
\left(\bar{v}_{0}-\bar{v}_{i}\right)^{2} \preceq \frac{H^{2}}{\left|\Omega_{0}\right|} \int_{\Omega_{0}}|\nabla v|^{2}+\frac{H^{2}}{\left|\Omega_{i}\right|} \int_{\Omega_{i}}|\nabla v|^{2}
$$

which together with (21) and a discrete Cauchy inequality give

$$
\begin{aligned}
\left(\eta_{0}\left|\Omega_{0}\right|\right. & \left.+\sum_{i=1}^{M} \eta_{i}\left|\Omega_{i}\right|\right)^{2} \bar{v}_{0}^{2}=\left(\sum_{i=1}^{M}\left(\bar{v}_{0}-\bar{v}_{i}\right) \eta_{i}\left|\Omega_{i}\right|\right)^{2} \\
& =\left(\sum_{i=1}^{M}\left(\bar{v}_{0}-\bar{v}_{i}\right) \sqrt{\eta_{i} \mid \Omega_{i}} \sqrt{\eta_{i}\left|\Omega_{i}\right|}\right)^{2} \\
& \preceq\left(\sum_{i=1}^{M} \eta_{i}\left|\Omega_{i}\right|\right) \sum_{i=1}^{M}\left(\bar{v}_{0}-\bar{v}_{i}\right)^{2} \eta_{i}\left|\Omega_{i}\right| \\
& \preceq\left(\sum_{i=1}^{M} \eta_{i}\left|\Omega_{i}\right|\right) \sum_{i=1}^{M} \eta_{i}\left|\Omega_{i}\right|\left(\frac{H_{0}^{2}}{\left|\Omega_{0}\right|} \int_{\Omega_{0}}|\nabla v|^{2}+\frac{H_{i}^{2}}{\left|\Omega_{i}\right|} \int_{\Omega_{i}}|\nabla v|^{2}\right) .
\end{aligned}
$$


Then

$$
\left(\beta_{0}+\sum_{i=1}^{M} \beta_{i}\right) \bar{v}_{0}^{2} \preceq\left(\sum_{i=1}^{M} \beta_{i}\right) \frac{H_{0}^{2}}{\left|\Omega_{0}\right|} \int_{\Omega_{0}}|\nabla v|^{2}+\sum_{i=1}^{M} \beta_{i} \frac{H_{i}^{2}}{\left|\Omega_{i}\right|} \int_{\Omega_{i}}|\nabla v|^{2}
$$

where, in order to simplify the notation, we defined

$$
\beta_{i}=\eta_{i}\left|\Omega_{i}\right|, \quad i=0,1, \ldots, M .
$$

We can bound

$$
\begin{aligned}
\eta_{0}\left|\Omega_{0}\right| \bar{v}_{0}^{2} \preceq & \frac{\sum_{i=1}^{M} \beta_{i}}{\beta_{0}+\sum_{i=1}^{M} \beta_{i}} \eta_{0} H_{0}^{2} \int_{\Omega_{0}}|\nabla v|^{2} \\
& +\sum_{i=1}^{M} \frac{\frac{\eta_{0}\left|\Omega_{0}\right|}{\eta_{i}\left|\Omega_{i}\right|} \beta_{i}}{\left(\beta_{0}+\sum_{i=1}^{M} \beta_{i}\right)^{2}} \eta_{i} H_{i}^{2} \int_{\Omega_{i}}|\nabla v|^{2} \\
\leq & \eta_{0} H_{0}^{2} \int_{\Omega_{0}}|\nabla v|^{2}+\sum_{i=1}^{M} \eta_{i} H_{i}^{2} \int_{\Omega_{i}}|\nabla v|^{2}
\end{aligned}
$$

where we have used that

$$
\frac{\sum_{i=1}^{M} \beta_{i}}{\beta_{0}+\sum_{i=1}^{M} \beta_{i}}<1
$$

and that

$$
\frac{\frac{\eta_{0}\left|\Omega_{0}\right|}{\eta_{i}\left|\Omega_{i}\right|} \beta_{i}}{\beta_{0}+\sum_{i=1}^{M} \beta_{i}}=\frac{\beta_{0}}{\beta_{0}+\sum_{i=1}^{M} \beta_{i}}<1
$$

On the other hand,

$$
\eta_{j}\left|\Omega_{j}\right| \bar{v}_{j}^{2} \leq \eta_{j}\left|\Omega_{j}\right| \bar{v}_{0}^{2}+\eta_{j}\left|\Omega_{j}\right|\left(\bar{v}_{j}-\bar{v}_{0}\right)^{2}
$$

Using (24) we have

$$
\begin{aligned}
\eta_{j}\left|\Omega_{j}\right| \bar{v}_{0}^{2} \preceq & \frac{\sum_{i=1}^{M} \beta_{i}}{\beta_{0}+\sum_{i=1}^{M} \beta_{i}} \frac{\eta_{j}\left|\Omega_{j}\right|}{\left|\Omega_{0}\right|} H^{2} \int_{\Omega_{0}}|\nabla v|^{2} \\
& +\sum_{i=1}^{M} \frac{\frac{\eta_{j}\left|\Omega_{j}\right|}{\eta_{i}\left|\Omega_{i}\right|} \beta_{i}}{\beta_{0}+\sum_{i=1}^{M} \beta_{i}} \eta_{i} H_{i}^{2} \int_{\Omega_{i}}|\nabla v|^{2} \\
\leq & \eta_{0} H_{0}^{2} \int_{\Omega_{0}}|\nabla v|^{2}+\sum_{i=1}^{M} \eta_{i} H_{i}^{2} \int_{\Omega_{i}}|\nabla v|^{2}
\end{aligned}
$$

where we have used the assumption $\frac{\eta_{i}\left|\Omega_{i}\right|}{\left|\Omega_{0}\right|} \preceq \eta_{0}, i=1,2, \ldots, M$ and

$$
\frac{\frac{\eta_{j}\left|\Omega_{j}\right|}{\eta_{i}\left|\Omega_{i}\right|} \beta_{i}}{\beta_{0}+\sum_{i=1}^{M} \beta_{i}}=\frac{\beta_{j}}{\beta+\sum_{i=1}^{M} \beta_{i}}<1
$$


Now, using (22) we can bound

$$
\begin{aligned}
\eta_{j}\left|\Omega_{j}\right|\left(\bar{v}_{j}-\bar{v}_{0}\right)^{2} & \preceq \eta_{j}\left|\Omega_{j}\right| \frac{H^{2}}{\left|\Omega_{0}\right|} \int_{\Omega_{0}}|\nabla v|^{2}+\eta_{j} H_{j}^{2} \int_{\Omega_{j}}|\nabla v|^{2} \\
& \preceq \eta_{0} H^{2} \int_{\Omega_{0}}|\nabla v|^{2}+\eta_{j} H_{j}^{2} \int_{\Omega_{j}}|\nabla v|^{2} .
\end{aligned}
$$

With (27) and (28) in (26) we get

$$
\eta_{j}\left|\Omega_{j}\right| \bar{v}_{j}^{2} \preceq \eta_{0} H_{0}^{2} \int_{\Omega_{0}}|\nabla v|^{2}+\sum_{i=1}^{M} \eta_{i} H_{i}^{2} \int_{\Omega_{i}}|\nabla v|^{2} .
$$

Finally, putting (29) and (25) in (20) we get,

$$
\begin{aligned}
\int_{\Omega} \kappa v^{2} & \preceq M \eta_{0} H_{0}^{2} \int_{\Omega_{0}}|\nabla v|^{2}+M \sum_{i=1}^{M} \eta_{i} H_{i}^{2} \int_{\Omega_{i}}|\nabla v|^{2} \\
& \preceq M H^{2} \int_{\Omega} \kappa|\nabla v|^{2} .
\end{aligned}
$$

Remark 6 We note that Lemma 5 holds for any inclusions $\Omega_{i}, i=1, \ldots, M$ that has Poincaré property (such that (19) and (18) hold). This also holds true for the case where some inclusions cross the boundary $\partial \Omega$.

Remark 7 In Lemma 5, $\kappa$ is assumed to be piecewise constants. These results can be extended to piecewise smooth coefficients such that the functions in each region $\Omega_{i}$ are of the form $\eta_{i} g_{i}(x)$ where $\left|g_{i}\right| \preceq 1$.

We need some estimates on the following quasi-interpolation operator. Define $I_{0}: V^{h} \rightarrow V^{H}$ by

$$
I_{0} v=\sum_{i=1}^{N_{c}} \bar{v}_{i} \Phi_{i} \quad \text { where } \quad \bar{v}_{i}=\frac{1}{\int_{\omega_{i}} \kappa} \int_{\omega_{i}} \kappa v .
$$

Here $\omega_{i}$ is the support of $\Phi_{i}$ (the union of coarse blocks with the node $x_{i}$ ). For each coarse block $K$ define $\omega_{K}=\cup_{i \in K} \omega_{i}$.

Lemma 8 Let the coefficient $\kappa$ be such that its restriction to $w_{K}$ satisfy the assumption of Lemma 5 for all coarse block $K$. Then for all $v \in V^{h}$ we have

$$
\int_{K} \kappa\left|v-I_{0} v\right|^{2} \leq C H^{2} \int_{\omega_{K}} \kappa|\nabla v|^{2}
$$

and

$$
\int_{K} \kappa\left|\nabla I_{0} v\right|^{2} \leq C \int_{\omega_{K}} \kappa|\nabla v|^{2}
$$


Proof. Recall that for each coarse basis functions we have $\left\|\Phi_{i}\right\|_{\infty} \preceq 1$. We also have for $\bar{v}_{i}$ defined in (32),

$$
\left|\bar{v}_{i}\right| \leq \frac{1}{\left(\int_{\omega_{i}} \kappa\right)^{\frac{1}{2}}}\left(\int_{\omega_{i}} \kappa v^{2}\right)^{\frac{1}{2}}
$$

and

$$
\begin{aligned}
\int_{K} \kappa\left|I_{0} v\right|^{2} & \leq \sum_{i=1}^{4}\left(\frac{1}{\int_{\omega_{i}} \kappa} \int_{\omega_{i}} \kappa v^{2}\right) \int_{K} \kappa\left|\Phi_{i}\right|^{2} \\
& \leq \sum_{i=1}^{4}\left(\frac{1}{\int_{\omega_{i}} \kappa} \int_{\omega_{i}} \kappa v^{2}\right) \int_{K} \kappa \\
& \leq C \int_{\omega_{K}} k v^{2} .
\end{aligned}
$$

Using this we obtain

$$
\int_{K} \kappa\left|v-I_{0} v\right|^{2} \leq 2 \int_{K} \kappa|v|^{2}+2 \int_{K} \kappa\left|I_{0} v\right|^{2} \leq C \int_{\omega_{K}} \kappa v^{2} .
$$

Then, if we put $\hat{v}=v-\frac{1}{\int_{\omega_{K}} \kappa} \int_{\omega_{K}} v$ we obtain

$$
\int_{K} \kappa\left|\hat{v}-I_{0} \hat{v}\right|^{2} \leq C \int_{\omega_{K}} \kappa \hat{v}^{2} \leq C H^{2} \int_{\omega_{K}} \kappa|\nabla v|^{2}
$$

where we have used Lemma 5 with $\Omega=w_{K}$. Now we prove (34). Using again Lemma 5 we obtain

$$
\begin{aligned}
\int_{K} \kappa\left|\nabla I_{0} v\right|^{2} & =\int_{K} \kappa\left|\nabla I_{0} \hat{v}\right|^{2} \\
& \leq \sum_{i=1}^{4}\left(\frac{1}{\int_{\omega_{i}} \kappa} \int_{\omega_{K}} \kappa \hat{v}^{2}\right) \int_{K} \kappa\left|\nabla \Phi_{i}\right|^{2} \\
& \leq \sum_{i=1}^{4}\left(\frac{1}{\int_{\omega_{i}} \kappa} \int_{\omega_{K}} \kappa \hat{v}^{2}\right) H_{K}^{-2} \int_{K} \kappa \\
& \preceq C H_{K}^{-2} \int_{\omega_{K}} k \hat{v}^{2} \\
& \preceq C \int_{\omega_{K}} k|\nabla v|^{2} .
\end{aligned}
$$

This finishes the proof.

Now, we highlight the differences between our analysis and the analysis presented in $[15,16]$; see also $[25,20]$. In the analysis presented in [15], the authors 
use standard volume averaging for $\overline{v_{i}}$. Thus, in the analysis, only standard Poincaré estimates are needed. In our analysis because of the weighted average definition of $\overline{v_{i}}$ in (32), we need to employ special Poincaré estimates. Note that the analysis of the cases considered here can not be carried out following the analysis presented in $[15,16]$.

Remark 9 One can choose the basis $\Phi_{i}$ such that $\int_{K} \kappa\left|\nabla \Phi_{i}\right|^{2} d x$ is minimized (as suggested in $[15,16]$ ), e.g., multiscale finite element basis functions or energy minimizing basis functions. It is evident from (35) that this will reduce the constant in (35) that directly affects the condition number; see Lemma 10 and Corollary 12 below.

\subsection{Analysis}

The analysis presented in this section is valid for coefficients that in each $w_{K}$ are as in in Lemma 5 (with $\Omega=w_{K}$ ). The analysis is also valid for more general coefficients; see Remark 7 and also Lemma 1 and Remarks 2, 3 and 4.

Lemma 10 Let $\kappa$ as in Lemma 5. For all $v \in V^{h}$, there exists a decomposition $v=\sum_{i=1}^{N} v_{i}$, with $v_{i} \in V_{0}^{i}\left(\Omega_{i}^{\prime}\right), i=0,1,2, \ldots$, such that

$$
a_{0}\left(v_{0}, v_{0}\right)+\sum_{i=1}^{N} a\left(v_{i}, v_{i}\right) \preceq C_{0}^{2} a(v, v) .
$$

where $C_{0}^{2} \preceq\left(\frac{H}{\delta}\right)^{2}$.

Proof. Define $v_{0}:=I_{0} v_{h}$ where $I_{0}$ is a coarse interpolation of Lemma 8 and

$$
v_{i}=\chi_{i}\left(v-v_{0}\right)
$$

where $\left\{\chi_{i}\right\}$ is a partition of unity subordinated to the overlapping partition $\left\{D_{i}^{\prime}\right\}$ and $\left|\nabla \chi_{i}\right| \leq \frac{1}{\delta}, i=1, \ldots, N$. First we bound the energy of $v_{i}$.

$$
\begin{aligned}
a\left(v_{i}, v_{i}\right) & =\int_{D_{i}^{\prime}} \kappa\left|\nabla\left(\chi_{i}\left(v-v_{0}\right)\right)\right|^{2} \\
& \preceq \int_{D_{i}^{\prime}} \kappa \chi_{i}^{2}\left|\nabla\left(v-v_{0}\right)\right|^{2}+\int_{D_{i}^{\prime}} \kappa\left|\nabla \chi_{i}^{2}\right|\left|v-v_{0}\right|^{2} \\
& \leq \int_{D_{i}^{\prime}} \kappa\left|\nabla\left(v-v_{0}\right)\right|^{2}+\frac{1}{\delta^{2}} \int_{D_{i}^{\prime} \backslash \overline{D_{i}}} \kappa\left|v-v_{0}\right|^{2} \\
& \preceq \int_{D_{i}^{\prime}} \kappa|\nabla v|^{2}+\int_{D_{i}^{\prime}} \kappa\left|\nabla v_{0}\right|^{2}+\frac{1}{\delta^{2}} \int_{D_{i}^{\prime} \backslash \overline{D_{i}}} \kappa\left|v-v_{0}\right|^{2} .
\end{aligned}
$$

Now we bound the last two terms of (40). 
The second term in (40) can be bounded using (34) of Lemma 8 as follows

$$
\int_{D_{i}^{\prime}} \kappa\left|\nabla v_{0}\right|^{2} \leq \sum_{\bar{K} \cap \bar{D}_{i} \neq \emptyset} \int_{K} \kappa\left|\nabla v_{0}\right|^{2} \leq C \sum_{K \cap \bar{D}_{i} \neq \emptyset} \int_{\omega_{K}} \kappa|\nabla v|^{2}
$$

where $\omega_{K}=\cup\left\{K^{\prime}: \bar{K} \cap \bar{K}^{\prime} \neq \emptyset\right\}$.

To bound the third term of (40) observe that in the case of generous overlap, i.e., $\frac{H}{\delta} \asymp C$, we have using (33) in Lemma 8

$$
\begin{aligned}
\frac{1}{\delta^{2}} \int_{D_{i}^{\prime} \backslash \overline{D_{i}}} \kappa\left|v-v_{0}\right|^{2} & \leq \frac{1}{\delta^{2}} \sum_{K \cap \bar{D}_{i} \neq \emptyset} \int_{K} \kappa\left|v-v_{0}\right|^{2} \\
& \leq C\left(\frac{H}{\delta}\right)^{2} \sum_{K \cap \bar{D}_{i} \neq \emptyset} \int_{\omega_{K}} \kappa|\nabla v|^{2} .
\end{aligned}
$$

The bound for the energy $a\left(v_{0}, v_{0}\right)$ follow from Lemma 8 .

Remark 11 In the proof of Lemma 10 we use the simple estimate (41). We avoid the use of bounds similar to those in [25, Lemma 3.10] (valid for bounded coefficients) or [15, Lemma 3.4] (valid when and special partition robustness indicator is bounded). Such bounds would improve the final condition number of the method.

We have the following bound form the condition number. See [25, 20].

Corollary 12 Under the assumptions of Lemma 10 the condition number of the preconditioned operator $B^{-1} A$ with $B^{-1}$ defined in (7) is of order

$$
\operatorname{cond}\left(B^{-1} A\right) \leq C\left(\frac{H}{\delta}\right)^{2}
$$

with $C$ independent of the contrast $\eta$.

With a generous overlap $\delta=c H$, we get $\operatorname{cond}\left(B^{-1} A\right) \leq C$.

Remark 13 From Lemma 5 we see that the constant of Corollary 12 depends on the number of inclusions. In the next section, we discuss how one can reduce the number of inclusions based on extension theorems.

\section{Overlapping methods for the Schur comple- ment}

In this section, we present analysis of overlapping methods for Schur complement; see [3] and references therein. These methods employ harmonic extensions and allow us to use extension theorems (e.g., $[14,9,10])$ to reduce the number of low conductivity inclusions. This reduction can be regarded as a partial homogenization. 


\subsection{Schur complement problem}

We define the local interfaces $\Gamma_{i}=\partial D_{i} \cap D, i=1, \ldots, N$ and the interface $\Gamma=\bigcup_{i=1}^{N} \Gamma_{i}$. Given $u \in V_{0}^{h}(D)$ we classify its degrees of freedom in

- $u_{\Gamma}$, the interface degrees of freedom, those that lies on $\Gamma$, and

- $u_{I}$, the interior degrees of freedom, those in the interior of any $D_{i}$ and outside $\Gamma$.

The $2 \times 2$ block structure of (4) is then

$$
\left[\begin{array}{ll}
A_{I I} & A_{I \Gamma} \\
A_{I \Gamma}^{T} & A_{\Gamma \Gamma}
\end{array}\right]\left[\begin{array}{l}
u_{I} \\
u_{\Gamma}
\end{array}\right]=\left[\begin{array}{l}
b_{I} \\
b_{\Gamma}
\end{array}\right]
$$

with analogous block structure of the local matrices $A^{(i)}, i=1, \ldots, N$ defined in $(5)$,

$$
\left[\begin{array}{cc}
A_{I I}^{(i)} & A_{I \Gamma}^{(i)} \\
A_{I \Gamma}^{(i) T} & A_{\Gamma \Gamma}^{(i)}
\end{array}\right] .
$$

For each $i, 1 \leq i \leq N$, we can consider $V_{0}^{h}\left(D_{i}\right)$ as a subset of the whole finite element space $V_{0}^{h}(D)$. We also define

$$
V_{0}^{h}(\Gamma)=\left\{v \in V_{0}^{h}(D): v(x)=0 \quad \text { for all vertex } x \notin \Gamma\right\} .
$$

Recall that $\kappa$ is the coefficient of the elliptic bilinear form $a$ defined in (2). Given $u \in V_{0}^{h}(D)$ define $\mathcal{P}_{\kappa} u \in V_{0}^{h}(\Gamma)$ as the $a$-orthogonal projection onto $V_{0}^{h}(\Gamma)$, is completely local and determined by

$$
a\left(\mathcal{P}_{\kappa} u, z\right)=a(u, z) \quad \text { for all } z \in V_{0}^{h}(\Gamma) .
$$

We also define the $\kappa$-harmonic extension $\mathcal{H}_{\kappa} u \in V^{h}(\Gamma)$ by 1 We define the set of $\kappa$-harmonic extensions by

$$
V^{h}(\Gamma)=\left\{v \in V_{0}^{h}(D): v=\mathcal{H}_{\kappa} v_{\Gamma}\right\}
$$

and the sets of local $\kappa$-harmonic extensions by

$$
V^{h}\left(\Gamma_{i}\right)=\left\{v \in V^{h}\left(D_{i}\right): a(v, z)=0 \text { for all } z \in V_{0}^{h}\left(D_{i}\right)\right\}, \quad i=1, \ldots, N .
$$

Let $u^{*}$ be the finite element solution of problem (3) then, $\mathcal{P}_{\kappa} u^{*}$ is obtained from

$$
a\left(\mathcal{P}_{\kappa} u^{*}, z\right)=f(z) \quad \text { for all } z \in V_{0}^{h}(\Gamma)
$$

which correspond to the solution of one Dirichlet problems per subdomain $D_{i}$. This computation can be done in parallel.

The component $\mathcal{H}_{\kappa}$ can be computed by solving the Schur complement problem

$$
a\left(\mathcal{H}_{\kappa} u, \mathcal{H}_{\kappa} v\right)=f\left(\mathcal{H}_{\kappa} v\right) \quad \text { for all } v \in V^{h}(\Gamma) .
$$


The matrix form of (45) is given by

$$
S u_{\Gamma}=\tilde{b}
$$

where

$$
S=A_{\Gamma \Gamma}-A_{I \Gamma}^{T} A_{I I}^{-1} A_{I \Gamma} \quad \text { and } \quad \tilde{b}=b_{\Gamma}-A_{I \Gamma}^{T} A_{I I}^{-1} b_{I} .
$$

We note that applying $A_{I I}^{-1}$ corresponds to the solution of $N$ uncoupled Dirichlet problems in each subdomain.

Define the local Schur complement bilinear form $s_{i}$ by

$$
s_{i}(u, v)=a_{i}\left(\mathcal{H}_{\kappa}^{(i)} u, \mathcal{H}_{\kappa}^{(i) v}\right) \quad \text { for all } u, v \in V^{h}\left(\Gamma_{i}\right), i=1, \ldots, N \text {; }
$$

where $\mathcal{H}_{\kappa}^{i}$ is the local $\kappa$-harmonic extension. The matrix form of the local Schur complement is given by

$$
S^{(i)}=A_{\Gamma \Gamma}^{(i)}-A_{I \Gamma}^{(i) T}\left(A_{I I}^{(i)}\right)^{-1} A_{I \Gamma}^{(i)} \quad i=1, \ldots, N .
$$

Then we can write

$$
S=\sum_{i=1}^{N} R_{i} S^{(i)} R_{i}^{T}
$$

where, for $i=1, \ldots, N, R_{i}: V^{h}\left(D_{i}\right) \cap V_{0}^{h}(D) \rightarrow V_{0}^{h}(D)$ is the extension by zero operator with a matrix which has only zeros and ones as entries.

Remark 14 We will solve the Schur complement system (45) or (46) using a preconditioned conjugate gradient method with two level Schwartz preconditioners. To apply $S$ we can use (47) which requires the solution of a local Dirichlet problem per subdomain.

\subsection{Preconditioner matrix form}

Consider an overlapping decomposition $\left\{D_{i}^{\prime}\right\}_{i=1}^{N^{\prime}}$. We assume that this decomposition is aligned with the coarse triangulation, i.e, each $D_{i}^{\prime}$ is the unions of coarse blocks. Put $\widetilde{\Gamma}_{i}=\Gamma \cap D_{i}^{\prime}$ Define the local spaces $V_{i}\left(\Gamma_{i}^{\prime}\right), i=1, \ldots, N^{\prime}$ as in (43) with $D_{i}^{\prime}$ instead of $D_{i}$. Let $\widetilde{S}_{i}$ be the Schur complement of $A_{i}$ defined in (8) with respect to $\Gamma_{i}^{\prime}$.

We use the following two level additive Schwartz preconditioners

$$
M^{-1}=R_{0} S_{0}^{-1} R_{0}+\sum_{i=1}^{N^{\prime}} R_{i}^{T} \widetilde{S}_{i}^{-1} R_{i}
$$

where $S_{0}=\left(R_{0} S R_{0}^{T}\right)^{-1}$ is the matrix form of $S$ on the coarse space $V_{0}$ define in (6) and $R_{0}^{T}$ is the projection on $V_{0}$.

Remark 15 Each application of $M^{-1}$ requires solving a coarse problem and a local problem per subdomain. 


\subsection{Preconditioner: condition number}

In Lemma 16 we concentrate on the dependence of the method on the heterogeneous coefficients.

Lemma 16 Let $\kappa$ be defined as in Lemma 10. For all $v \in V^{h}$, there exists a decomposition $v=\sum_{i}^{N} v_{i}$, with $v_{i} \in V_{0}^{i}\left(\Gamma_{i}^{\prime}\right), i=0,1,2, \ldots$, such that

$$
s_{0}\left(v_{0}, v_{0}\right)+\sum_{i=1}^{N} s\left(v_{i}, v_{i}\right) \preceq C_{0}^{2} s(v, v)
$$

where $C_{0}^{2}$ is independent of the contrast $\eta$.

Proof. Define $v_{0}:=I_{0} \mathcal{H}_{\kappa} v_{h}$ where $I_{0}$ is a coarse interpolation of Lemma 8 and

$$
v_{i}=\chi_{i}\left(v-v_{0}\right) \quad \text { on } \Gamma_{i}^{\prime}
$$

where $\left\{\chi_{i}\right\}$ is a partition of unity subordinated to the overlapping partition $\left\{D_{i}^{\prime}\right\}$ with $\left|\chi_{i}\right| \leq \frac{1}{H}, i=1, \ldots, N^{\prime}$. First we bound the energy of $v_{i}$.

$$
\begin{aligned}
s\left(v_{i}, v_{i}\right) & =\int_{D_{i}^{\prime}} \kappa\left|\nabla \mathcal{H}_{\kappa}\left(\chi_{i}\left(v-v_{0}\right)\right)\right|^{2} \leq \int_{D_{i}^{\prime}} \kappa\left|\nabla \chi_{i} \mathcal{H}_{\kappa}\left(v-v_{0}\right)\right|^{2} \\
& \preceq \int_{D_{i}^{\prime}} \kappa \chi_{i}^{2}\left|\nabla \mathcal{H}_{\kappa}\left(v-v_{0}\right)\right|^{2}+\int_{D_{i}^{\prime}} \kappa\left|\nabla \chi_{i}\right|^{2}\left|\mathcal{H}_{\kappa}\left(v-v_{0}\right)\right|^{2} \\
& \leq \int_{D_{i}^{\prime}} \kappa\left|\nabla \mathcal{H}_{\kappa}\left(v-v_{0}\right)\right|^{2}+\frac{1}{H^{2}} \int_{D_{i}^{\prime} \backslash \overline{D_{i}}} \kappa\left|\mathcal{H}_{\kappa}\left(v-v_{0}\right)\right|^{2} \\
& \preceq \int_{D_{i}^{\prime}} \kappa\left|\nabla \mathcal{H}_{\kappa} v\right|^{2}+\int_{D_{i}^{\prime}} \kappa\left|\nabla \mathcal{H}_{\kappa} v_{0}\right|^{2}+\frac{1}{H^{2}} \int_{D_{i}^{\prime} \backslash \overline{D_{i}}} \kappa\left|\mathcal{H}_{\kappa}\left(v-v_{0}\right)\right|^{2} .
\end{aligned}
$$

Now we bound the last two terms of (49).

The second term in (49) can be bounded using (34) of Lemma 8 as follows

$$
\int_{D_{i}^{\prime}} \kappa\left|\nabla \mathcal{H}_{\kappa} v_{0}\right|^{2} \leq \sum_{\bar{K} \cap \bar{D}_{i} \neq \emptyset} \int_{K} \kappa\left|\nabla v_{0}\right|^{2} \leq C \sum_{K \cap \bar{D}_{i} \neq \emptyset} \int_{\omega_{K}} \kappa\left|\nabla \mathcal{H}_{\kappa} v\right|^{2}
$$

where $\omega_{K}=\cup\left\{K^{\prime}: \bar{K} \cap \bar{K}^{\prime} \neq \emptyset\right\}$.

To bound the third term of (49) observe that, (33) in Lemma 8

$$
\begin{aligned}
\frac{1}{H^{2}} \int_{D_{i}^{\prime} \backslash \overline{D_{i}}} \kappa\left|\mathcal{H}_{\kappa}\left(v-v_{0}\right)\right|^{2} & \leq \frac{1}{H^{2}} \sum_{K \cap \bar{D}_{i} \neq \emptyset} \int_{K} \kappa\left|\mathcal{H}_{\kappa} v-v_{0}\right|^{2} \\
& \leq C \sum_{K \cap \bar{D}_{i} \neq \emptyset} \int_{\omega_{K}} \kappa\left|\nabla \mathcal{H}_{\kappa} v\right|^{2} .
\end{aligned}
$$

The bound for the energy $s\left(v_{0}, v_{0}\right)$ follows from Lemma 8 . 
Corollary 17 Under the assumption of Lemma 16, the condition number of the preconditioned operator $M^{-1} S$ with $M^{-1}$ defined in (48) is bounded, i.e., cond $\left(M^{-1} S\right) \leq C$ with $C$ independent of the contrast $\eta$.

\subsection{Reducing the number of inclusions - partial energy homogenization}

Previous analysis assumes that the number of inclusions is finite. The number of inclusions directly affects the number of iterations in domain decomposition methods as it is evident from Lemma 5. In many applications, the number of inclusions can be very large introducing a small scale into the problem. One can reduce the number of inclusions by using extension theorems (e.g., $[14,9,10])$. These theorems are often used in homogenization. This reduction can be regarded as a partial homogenization because it homogenizes many inclusions such that the resulting media have fewer inclusions and have an equivalent energy as the original media. This type of homogenization is possible within the Schur complement framework. We note that a difference from the homogenization is that the equivalent energy is sought for arbitrary boundary conditions here.

Next, we briefly describe it. Assume the domain $\Omega$ contains many inclusions with lower conductivity and denote these regions by $\Omega_{1}$. As before $\Omega=\Omega_{\eta} \bigcup \Omega_{1}$ where $\Omega_{1}$ denotes the union of domains that have conductivity 1 . We call the media $E_{\preceq}$ type if for any function $v \in H^{1}\left(\Omega_{\eta}\right)$, there exists an extension $v_{e}$ such that

$$
\int_{\Omega}\left|\nabla v_{e}\right|^{2} \preceq \int_{\Omega_{\eta}}|\nabla v|^{2} .
$$

This holds for any inclusion $\Omega_{1}$ where $\Omega_{\eta}$ is a connected region [14]. It also holds for inclusions that are periodic or randomly distributed $([10,27])$.

Next, we demonstrate how the extension theorem can be used to prove the energy equivalence. First, we show that if the domain is type $E_{\preceq}$ then

$$
\int_{\Omega} \kappa^{*}\left|\nabla \mathcal{H}_{\kappa^{*}} \mu\right|^{2} \preceq \int_{\Omega} \kappa\left|\nabla \mathcal{H}_{\kappa} \mu\right|^{2} \preceq \int_{\Omega} \kappa^{*}\left|\nabla \mathcal{H}_{\kappa^{*}} \mu\right|^{2} \quad \text { for all } \mu \in H^{1 / 2}(\partial \Omega),
$$

where $\kappa^{*}=\eta$ is the homogenized coefficient. For the upper bound, we have

$$
\int_{\Omega} \kappa\left|\nabla \mathcal{H}_{\kappa} \mu\right|^{2} \preceq \int_{\Omega} \kappa\left|\nabla \mathcal{H}_{\kappa^{*}} \mu\right|^{2} \preceq \int_{\Omega} \kappa^{*}\left|\nabla \mathcal{H}_{\kappa^{*}} \mu\right|^{2} .
$$

For the first inequality, we have used the fact that the energy is the lowest for the harmonic extension, while for the second inequality, we have used the fact that $\kappa \preceq \kappa^{*}$. For the lower bound, we have

$$
\int_{\Omega} \kappa^{*}\left|\nabla \mathcal{H}_{\kappa^{*}} \mu\right|^{2} \preceq \int_{\Omega} \kappa^{*}\left|\nabla v_{e}\right|^{2} \preceq \int_{\Omega_{\eta}} \kappa^{*}\left|\nabla \mathcal{H}_{\kappa} \mu\right|^{2} \preceq \int_{\Omega} \kappa\left|\nabla \mathcal{H}_{\kappa} \mu\right|^{2}
$$

where $v_{e}$ is the function that is equal to $v=H_{\kappa} \mu$ in $\Omega_{\eta}$ and its $E_{\prec}$ extension in $\Omega_{1}$. For the second inequality, we have used the extension estimate formulated earlier. 


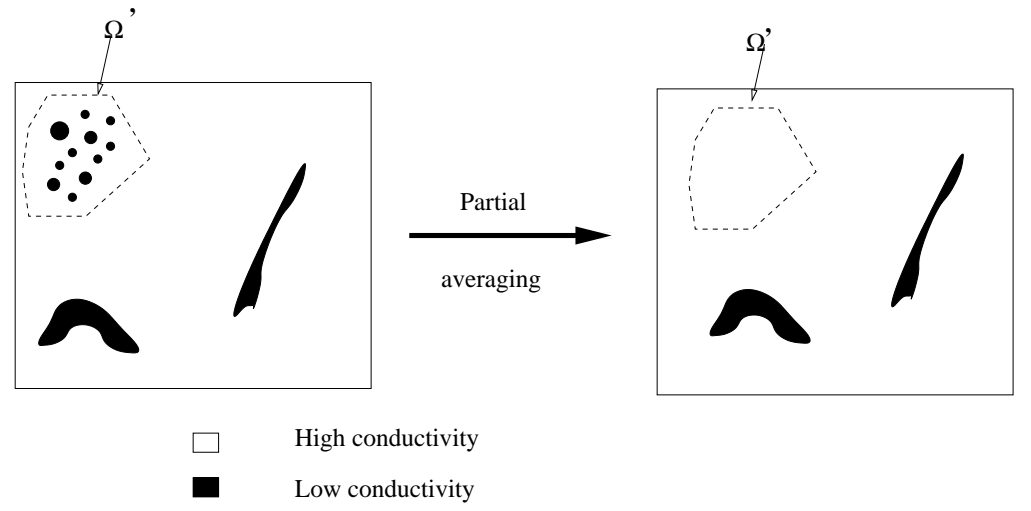

Figure 4:

Next, we present the result for more general case (see Fig. 4). In particular, we assume that $\Omega^{\prime} \subset \Omega$ contains inclusions that can be homogenized using the extension theorem, while the rest of the domain $\Omega-\Omega^{\prime}$ remains unchanged. By homogenizing a part of the region, we can lower the number of inclusions significantly, in general.

The estimate for the upper bound is the same as before:

$$
\int_{\Omega} \kappa^{*}\left|\nabla \mathcal{H}_{\kappa^{*}} \mu\right|^{2} \preceq \int_{\Omega} \kappa\left|\nabla \mathcal{H}_{\kappa} \mu\right|^{2} \preceq \int_{\Omega} \kappa^{*}\left|\nabla \mathcal{H}_{\kappa^{*}} \mu\right|^{2} .
$$

For the lower bound, we first use the variational principle and replace $\mathcal{H}_{\kappa^{*}} \mu$ by $v_{e}$ and then divide the integral into two parts

$$
\int_{\Omega} \kappa^{*}\left|\nabla \mathcal{H}_{\kappa^{*}} \mu\right|^{2} \preceq \int_{\Omega^{\prime}} \kappa^{*}\left|\nabla v_{e}\right|^{2}+\int_{\Omega-\Omega^{\prime}} \kappa^{*}\left|\nabla v_{e}\right|^{2}
$$

where $v_{e}=v=\mathcal{H}_{\kappa} \mu$ in $\Omega_{\eta} \bigcap \Omega$ and $E_{\preceq}$ type extension in $\left(\Omega_{1} \bigcap \Omega^{\prime}\right) \bigcap \Omega$. Then

$$
\int_{\Omega^{\prime}} \kappa^{*}\left|\nabla v_{e}\right|^{2} \preceq \int_{\Omega^{\prime} \cap \Omega_{\eta}} \kappa^{*}\left|\nabla v_{e}\right|^{2}=\int_{\Omega^{\prime} \cap \Omega_{\eta}} \kappa|\nabla v|^{2} \preceq \int_{\Omega^{\prime}} \kappa|\nabla v|^{2} .
$$

Further we note that

$$
\int_{\Omega-\Omega^{\prime}} \kappa^{*}\left|\nabla v_{e}\right|^{2}=\int_{\Omega-\Omega^{\prime}} \kappa^{*}|\nabla v|^{2}
$$

Remark 18 The equivalence of energies can be easily used in Lemma 16. We can replace $k$ by $k^{*}$. Then, the condition number of the preconditioned Schur complement $M^{-1} S$ with preconditioner $M^{-1}$ defined in (48) will depend only on the number of inclusions of $\kappa^{*}$. 


\section{A comment on multi-phase flow equations}

In this section, we comment on the use of domain decomposition methods in multi-phase flow. Two-phase flow equations described by

$$
\operatorname{div}(\lambda(S) \kappa \nabla p)=f
$$

in the absence of gravity and capillary effects. The total mobility $\lambda(S)$ is given by $\lambda(S)=\lambda_{w}(S)+\lambda_{o}(S)$ and $f$ is a source term. Here, $\lambda_{w}(S)=k_{r w}(S) / \mu_{w}$ and $\lambda_{o}(S)=k_{r o}(S) / \mu_{o}$ where $\mu_{o}$ and $\mu_{w}$ are viscosities of oil and water phases, correspondingly, and $k_{r w}(S)$ and $k_{r o}(S)$ are relative permeabilities of oil and water phases, correspondingly. The transport equation is governed by

$$
\frac{\partial S}{\partial t}+\operatorname{div}(F)=0
$$

where $F=v f_{w}(S)$, with $f_{w}(S)$, the fractional flow of water, given by $f_{w}=$ $\lambda_{w} /\left(\lambda_{w}+\lambda_{o}\right)$, and the total velocity $v$ by:

$$
v=v_{w}+v_{o}=-\lambda(S) \kappa \nabla p
$$

The equation (50) is solved repeatedly for different $\lambda(S)$. Typically, $\kappa(x)$ has a high contrast as discussed earlier. If $\lambda(S)$ is bounded, it can be easily shown that the preconditioners discussed earlier for $\lambda=1$ can be used for preconditioning the discrete system corresponding to (50). More precisely, one can show that the condition number of the preconditioned system is independent of the contrast in $\kappa$.

\section{Numerical experiments}

In this section, we present representative numerical results for the overlapping methods. Various heterogeneities are considered in the simulations. We show that the condition number of the resulting preconditioned system is independent of the contrast as our theory shows.

We take $D=[0,1] \times[0,1]$ divided in $10 \times 10$ subdomains. Inside each subdomain we use a fine scale triangulation with $h=1 / 100$. We consider three types of coefficients

1. High conductivity background with low conductivity inclusions.

2. High conductivity background with low conductivity channels.

3. Mixture of piecewise Gaussian permeability fields (generated using truncated Karhunen-Loève [18] expansions) with highly varying means and synthetic inclusions.

We solve the equation $-\operatorname{div}(\kappa \nabla u)=0$ with $u\left(x_{1}, x_{2}\right)=x_{1}$ on $\partial D$. We run the Preconditioned Conjugate Gradient (PCG) until the $\ell_{2}$ norm of the residual 
is reduced by a factor of $10^{6}$. We will present the number of iterations until the convergence of the PCG because this quantity is a main factor in determining the efficiency of the preconditioner. We have also tested our methods for $-\operatorname{div}(\kappa \nabla u)=f$ in $D$ and $u=0$ on $\partial D$ and observed similar results, i.e., preconditioned system is independent of the contrast.

We note that for the Type 3 coefficients and our choice of the coarse-grid blocks there are a few coarse blocks where the assumption on the form of the coefficient $\kappa$ may not hold; however, we still observe good performance of the preconditioner for this case. More detailed numerical studies for various heterogeneities that arise in petroleum applications will be presented elsewhere.

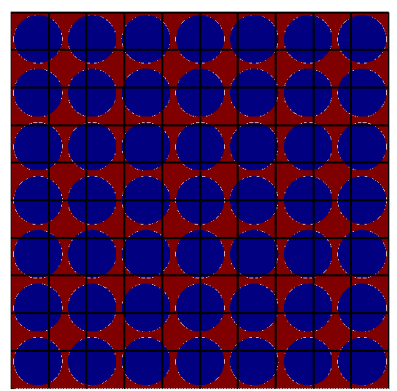

Figure 5: Type 1 coefficients and a coarse triangulation. Red designates the regions where the coefficient is $\eta$ and blue designates the regions where the coefficient is 1 . The numerical results are presented in Table 1. Note that the coarse triangulation is not aligned with the inclusions.

For our first numerical example, we consider the coefficients as indicated in Type 1, see Figure 5. We test different values for the contrast. In Table 1 we show the iteration count for the two level additive methods for (4) and (46). We tested different coarse basis: piecewise linear, multiscale basis and energy minimizing functions. Note that the coarse triangulation is not aligned with the inclusions in the numerical test. We observe from this table that the condition number of the resulting preconditioned system is independent of the contrast. We observe slight improvement when multiscale and energy minimizing basis functions are used for the coarse-scale approximation.

We repeat the numerical experiment for the Type 2 coefficients as plotted in Figure 6. The results are shown in Table 2. We note that the coarse mesh is again not aligned with the boundaries of low conductivity regions. As before, we observe that the condition number of the resulting preconditioned system is independent of the contrast. We observe slight improvement when multiscale basis functions are used for the coarse-scale approximation.

We finally test the two level additive preconditioners with the Type 3 coefficients as depicted in Figure 7. The permeability field is created using three independent realizations of log-Gaussian fields generated via truncated KarhunenLoève [18]. In the upper part, a realization of a log-Gaussian field with cor- 


\begin{tabular}{|l|c|c|c|}
\hline$\eta$ & Linear & MS & EMF \\
\hline 1 & 13 & $13(12)$ & $18(16)$ \\
10 & 18 & $15(13)$ & $21(18)$ \\
100 & 21 & $17(14)$ & $22(19)$ \\
1000 & 21 & $18(15)$ & $22(19)$ \\
10000 & 21 & $18(15)$ & $23(19)$ \\
100000 & 21 & $18(15)$ & $23(19)$ \\
1000000 & 22 & $18(15)$ & $23(19)$ \\
\hline \hline
\end{tabular}

Table 1: Number of iteration until convergence of the PCG for different values of the contrast $\eta$ with Type 1 coefficients; see Figure 5. We solve (4) (resp. (46)) with preconditioner $B^{-1}$ in (7) (resp. $M^{-1}$ in (48)). Different coarse problems: piecewise linear (Linear), multiscale (MS) and energy minimizing functions (EM). Here we have $H=1 / 10, h=1 / 100$ and $\delta=H$.

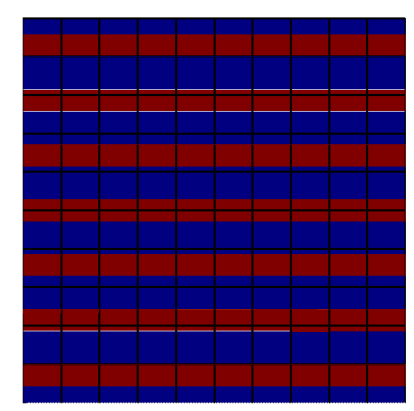

Figure 6: Coefficient and coarse triangulation. Red designates the regions where the coefficient is $\eta$ and blue designates the regions where the coefficient is 1 . Numerical results are presented in Table 2.

relation lengths $L_{1}=L_{2}=0.1$ and zero mean is used. In the middle part, a realization of a log-Gaussian field with correlation lengths $L_{1}=0.4$ and $L_{2}=0.1$ and mean $7+\eta$ (that is varied) is used. In the lower part of the domain, a realization of a log-Gaussian field with correlation lengths $L_{1}=0.3$ and $L_{2}=0.1$ and zero mean is used. Further, we include low contrast inclusions in the middle part (high contrast part) of the domain as depicted in Figure 7. We have tested our methods with inclusions of various shapes and geometries. Here, we only restrict ourselves to one of such cases. Numerical results presented in Table 3 demonstrate that the condition number of the resulting preconditioned system is independent of the contrast as predicted by the theory. 


\begin{tabular}{|l|c|c|c|}
\hline$\eta \downarrow$ & Linear & MS & EM \\
\hline 1 & 13 & $13(12)$ & $18(16)$ \\
10 & 19 & $15(17)$ & $18(18)$ \\
100 & 22 & $16(23)$ & $20(20)$ \\
1000 & 21 & $17(28)$ & $19(22)$ \\
10000 & 22 & $17(28)$ & $19(22)$ \\
100000 & 22 & $17(28)$ & $19(22)$ \\
\hline \hline
\end{tabular}

Table 2: Number of iteration for different values of the contrast $\eta$ with Type 2 coefficient. See Figure 6 . Here we have $H=1 / 10$ and $h=1 / 100$.

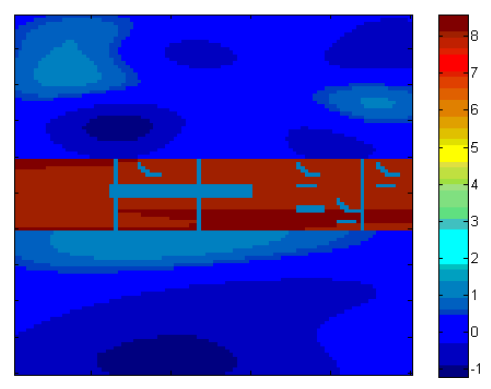

Figure 7: Mixture of log-Gaussian permeabilities with different means. Numerical results are presented in in Table 3.

Remark 19 (Nonoverlapping methods) In Table 4 we present numerical results for nonoverlapping domain decomposition methods for the Schur complement problem. We use a two level additive method; see [25, 20]. These numerical results show that the condition number of the resulting preconditioned system for the Type 3 coefficient is nearly independent of the contrast. However, we observe that the number of iterations in the nonoverlapping case is larger than that in the overlapping case. The condition number for this method if of order $(1+\log (H / h))^{2}$ when using special coarse solvers [20, 25] in the case of piecewise constant coefficients. The construction of the preconditioner relies on the fact that the coefficients are scaled uniformly in each coarse block (i.e., they are either high or bounded). The extension of these results to the case of highly variable coefficients is not straightforward and will be object of future work.

\section{Conclusions}

In this paper we study preconditioners for multiscale elliptic problems with highly variable coefficients. We analyze theoretically and numerically two level additive Schwartz preconditioners with several coarse solvers. Our objective is to show that the condition number of preconditioned systems is independent of 


\begin{tabular}{|c|c|c|c|}
\hline$\eta$ & Linear & MS & EMF \\
\hline 1 & 32 & $27(27)$ & $22(23)$ \\
2 & 35 & $29(29)$ & $28(28)$ \\
3 & 37 & $31(32)$ & $30(31)$ \\
4 & 39 & $33(33)$ & $33(34)$ \\
5 & 41 & $35(35)$ & $35(35)$ \\
6 & 33 & $28(37)$ & $37(38)$ \\
7 & 34 & $29(29)$ & $40(40)$ \\
\hline \hline
\end{tabular}

Table 3: Number of iteration for different values of the mean of the middle part of the Type 3 coefficient which is a mixture of log-Gaussian permeabilities with different means. See Figure 7 for the coefficient with $\eta=0$. Here we have $H=1 / 10$ and $h=1 / 100$.

\begin{tabular}{|r|r|r|}
\hline$\eta$ & MS & EMF \\
\hline 1 & 80 & 69 \\
2 & 85 & 80 \\
3 & 87 & 102 \\
4 & 129 & 131 \\
5 & 123 & 154 \\
6 & 140 & 182 \\
7 & 157 & 217 \\
\hline \hline
\end{tabular}

Table 4: Number of iteration for different values of the mean of the middle part of the Type 3 which is a mixture of log-Gaussian permeabilities with different means. See Figure 7 for the coefficient with $\eta=0$. We solve (46) with a two level additive nonoverlapping method; see [20]. Here we have $H=1 / 10$ and $h=1 / 100$.

the contrast in the media properties. In the overlapping setting, we concentrate on the case of a generous overlap. In our analysis, we define a coarse-scale approximation using weighted averages. The weighted averages are important to show $L_{2}$ approximation in the analysis. Our analysis requires special Poincaré estimates to bound the weighted $L_{2}$ estimates. We study the heterogeneities when the low conductivity regions are embedded into high conductivity regions. We also study overlapping methods for Schur complement methods. These methods employ harmonic extensions and allow us to use extension theorems to reduce the number of low conductivity inclusions. In the paper, we present numerical results that confirm our theoretical results.

\section{References}

[1] J. E. Aarnes, On the use of a mixed multiscale finite element method for greater flexibility and increased speed or improved accuracy in reservoir sim- 
ulation, SIAM MMS, 2 (2004), pp. 421-439.

[2] J. E. Aarnes, Y. Efendiev, and L. Jiang, Analysis of Multiscale Finite Element Methods using Global Information For Two-Phase Flow Simulations, SIAM MMS, 2008.

[3] J Aarnes and T Hou, Multiscale domain decomposition methods for elliptic problems with high aspect ratios, Acta Math. Appl. Sin. Engl. Ser., 18(1):63$76,2002$.

[4] J. E. Aarnes, S. Krogstad, and K.-A. Lie, A hierarchical multiscale method for two-phase flow based upon mixed finite elements and nonuniform grids, Multiscale Model. Simul. 5(2) (2006), pp. 337-363.

[5] T. Arbogast, Implementation of a locally conservative numerical subgrid upscaling scheme for two-phase Darcy flow, Comput. Geosci., 6 (2002), pp. 453-481. Locally conservative numerical methods for flow in porous media.

[6] T. Arbogast, G. Pencheva, M. F. Wheeler, and I. Yotov, A multiscale mortar mixed finite element method, SIAM J. Multiscale Modeling and Simulation, 6(1), 2007, pp. 319-346

[7] Y. Chen, L. J. Durlofsky, M. Gerritsen, and X. H. Wen, A coupled local-global upscaling approach for simulating flow in highly heterogeneous formations, Advances in Water Resources, 26 (2003), pp. 1041-1060.

[8] Z. Chen and T. Y. Hou, A mixed multiscale finite element method for elliptic problems with oscillating coefficients, Math. Comp., 72 (2002), pp. 541-576 (electronic).

[9] D. Cioranescu and J. Saint Jean Paulin, Homogenization in Open Sets with Holes, J. Math. Anal. Appl. 71 (2), 590607 (1979).

[10] A. Damlamian and P. Donato, Which sequences of holes are admissible for periodic homogenization with Neumann boundary condition?, ESAIM: Control, Optimisation and Calculus of Variations, 8 (2002), p. 555-585

[11] Y. Efendiev, V. Ginting, T. Hou, and R. Ewing, Accurate multiscale finite element methods for two-phase flow simulations, J. Comp. Physics, 220 (1), pp. 155-174, 2006.

[12] Y. Efendiev, T. Hou, and V. Ginting, Multiscale finite element methods for nonlinear problems and their applications, Comm. Math. Sci., 2 (2004), pp. $553-589$.

[13] Y. Efendiev, T. Y. Hou, and X. H. Wu, Convergence of a nonconforming multiscale finite element method, SIAM J. Num. Anal., 37 (2000), pp. 888910. 
[14] V. Jikov, S. Kozlov, and O. Oleinik. Homogenization of differential operators and integral functionals, Springer-Verlag, 1994, Translated from Russian.

[15] I. G. Graham, P. O. Lechner, and R. Scheichl, Domain decomposition for multiscale PDEs. Numer. Math., 106(4):589-626, 2007.

[16] I. G. Graham and R. Scheichl, Robust domain decomposition algorithms for multiscale PDEs, Numer. Methods Partial Differential Equations, 23(4):859$878,2007$.

[17] H. Li and C. D. White, Geostatistical models for shales in distributary channel point bars (Ferron Sandstone, Utah): From ground-penetrating radar data to three-dimensional flow modeling, American Association of Petroleum Geologists (AAPG), ecember 2003; v. 87; no. 12; p. 1851-1868; DOI: $10.1306 / 07170302044$

[18] M. Loève. Probability theory. 4th ed., Springer, Berlin, 1977.

[19] I. Lunati and P. Jenny, Multi-scale finite-volume method for highly heterogeneous porous media with shale layers, in: Proceedings of the 9th European Conference on the Mathematics of Oil Recovery (ECMOR), Cannes, France, 2004.

[20] Tarek P. A. Mathew. Domain decomposition methods for the numerical solution of partial differential equations, volume 61 of Lecture Notes in Computational Science and Engineering. Springer-Verlag, Berlin, 2008.

[21] T. Y. Hou and X. H. Wu, A multiscale finite element method for elliptic problems in composite materials and porous media, Journal of Computational Physics, 134 (1997), pp. 169-189.

[22] T. Hughes, G. Feijoo, L. Mazzei, and J. Quincy, The variational multiscale method - a paradigm for computational mechanics, Comput. Methods Appl. Mech. Engrg, 166 (1998), pp. 3-24.

[23] P. Jenny, S. H. Lee, and H. Tchelepi, Multi-scale finite volume method for elliptic problems in subsurface flow simulation, J. Comput. Phys., 187 (2003), pp. 47-67.

[24] H. Owhadi and L. Zhang, Metric based up-scaling, Comm. Pure Appl. Math., 2007.

[25] Andrea Toselli and Olof Widlund. Domain decomposition methodsalgorithms and theory, volume 34 of Springer Series in Computational Mathematics. Springer-Verlag, Berlin, 2005.

[26] Jinchao Xu and Jun Zou. Some nonoverlapping domain decomposition methods. SIAM Rev., 40(4):857-914 (electronic), 1998. 
[27] V.V. Zhikov, Averaging in perforated random domains of general type, Matematicheskie Zametki, Vol. 53, No. 1, pp. 4158, January, 1993. 